\title{
COMMISSION 35: STELLAR CONSTITUTION (CONSTITUTION DES ÉTOILES)
}

Report 1993-1996

President: C. Chiosi

Vice-President: J.-P. Zahn

Organizing Committee: P. Demarque (past president), I. Iben Jr., A. Maeder, G. Michaud, K. Nomoto, A. Renzini, V. Tarady, A.V. Tutukov, D. VandenBerg

\section{Introduction}

This report focuses on a few subjects where substantial progress has been achieved during the past three years. Several colleagues were asked to describe what they consider as the highlights in their field, and we are very grateful to them for their prompt and competent response. They covered the following topics: massive stars (A. Maeder), intermediate mass stars (P. Demarque), low mass objects (G. Chabrier), stellar convection (H. Spruit), atomic diffusion (G. Michaud), rotational mixing (J.-P. Zahn), helioseismology (S. Basu) and solar neutrinos (E. Schatzman). J.-P. Zahn was responsible for collecting and editing their contributions.

\section{Meetings, Symposia, Colloquia}

\subsection{OF DIRECT INTEREST TO COMMISSION 35}

IAU Symposium 162, Pulsation, Rotation and Mass Loss in Early-type Stars, Juan-les-Pins (France), Oct. 1993, ed. L.A. Balona, H.F. Henrichs \& J.M. Le Contel, Kluwer.

IAU Symposium 181, Sounding Solar and Stellar Interiors, Nice (France), Sept. 1996, ed. G. Berthomieu \& F.X. Schmider, Kluwer.

IAU Colloquium 147, The Equation of State in Astrophysics, St Malo (France), June 1993, ed. G. Chabrier \& E. Schatzman, Cambridge University Press.

Binaries as Tracers of Stellar Formation, Bettmeralp (Switzerland), Sept. 1991, ed. A. Duquennoy \& M. Mayor, Cambridge University Press.

Evolution of Massive Stars: A Confrontation between Theory and Observation, Brussels (Belgium), Aug. 1993, ed. D. Vanbeveren, W. van Rensbergen \& C. de Loore, Kluwer.

Stellar and Interstellar Lithium and Primordial Nucleosynthesis, The Hague (Netherlands), Aug. 94, Mem. Soc. Astr. Ital. 66, 307.

32nd Liège International Colloquium, Stellar Evolution: What should be done, July 1995, ed. A. Noels, D. Fraipont-Caro, M. Gabriel, N. Grevesse \& P. Demarque.

8th Cambridge Workshop on Cool Stars, Stellar Systems and the Sun, Athens (USA), Oct. 1995, , ed. J.P. Caillault, ASP conf. ser. 64. 
9th Cambridge Workshop on Cool Stars, Stellar Systems and the Sun, Oct. 1995, Florence (Italy).

Solar Convection and Oscillations and their Relationship, Aarhus (Denmark), May 1996, ed. F. Pijpers (Kluwer).

\subsection{OTHER MEETINGS OF INTEREST}

IAU Symposium 143, The Sun as a Variable Star: Solar \& Stellar Irradiance Variations, Boulder (USA), June 1993, ed. J.M. Pap, C. Fröhlich, H. S. Hudson \& K. Solanki, Cambridge University Press.

IAU Symposium 165, Compact Stars in Binaries, The Hague (Netherlands), Aug. 1993, ed. J. van Paradijs, E.J. van den Heuvel \& E. Kuulkers, Kluwer.

IAU Symposium 166, Astronomical and Astrophysical Objectives of Sub-milliarcsecond Optical Astrometry, The Hague (Netherlands), Aug. 1994, ed. Høg \& Seidelmann, Kluwer.

IAU Symposium 176, Stellar Surface Structure, Vienna (Austria), Oct. 1995.

IAU Symposium 177, The carbon Star Phenomenon, Antalya (Turkey), May 1996.

IAU Colloquium 155, Astrophysical Applications of Stellar Pulsation, Cape Town, South Africa, Feb. 1995, ed. R.S. Stobie \& P.A. Whitelock, ASP conf. ser. 83.

IAU Colloquium 158, Cataclysmic Variables and Related Objects, Keele (UK), June 1995.

GONG '94: Helio- and astero-seismology from the Earth and Space, Los Angeles (USA), May 1994, ed. R.J. Ulrich, E.J. Rhodes Jr. \& W. Däppen, ASP conf. ser. 76.

XVIIth International Conference on Neutrino Physics and Astrophysics, Helsinki (Finland), June 1996.

32nd Liège International Colloquium, Wolf-Rayet Stars in the Framework of Stellar Evolution, July 1996.

Variable Stars and the Astrophysical Return of Microlensing, Paris (France), July 1996.

\section{Highlights}

\subsection{MASSIVE STARS}

The field of massive star evolution has received during the last years a strong impetus from many new observations on surface chemical abundances, stellar winds, massive star populations in galaxies, starbursts, supernova progenitors, chemical evolution of galaxies, etc.. THE HOT STAR NEwSLETTER published monthly by Philippe Eenens (email:eenens@inaoep.mx) has become a major source of information in the field.

\subsubsection{Pre-Main Sequence Evolution}

The difficulty to match the observations of T-Tauri stars and Ae Herbig stars by standard pre-MS evolutionary tracks, and the rather general observation of accretion disks around young stars strongly support the new accretionscenario (Palla \& Stahler 1992, ApJ 392, 667; 1993, ApJ 418, 414). The accreting models initially follow in the HR diagram a common ascending evolutionary path, called the birth line, from which they deviate when the accretion rate becomes negligible. Only a few works have been devoted to the pre-MS evolution of massive stars (Beech \& Mitalas, 1994, ApJ 95, 517; Bernasconi \& Maeder, 1996, A\&A 307, 829). Drastically new results are coming out: the stars during their accretion phase are nearly raising up the zero age sequence, the pre-MS lifetime of a $40 \mathrm{M}_{\odot}$ star is about $10^{6} \mathrm{yr}$, rather than a few $10^{4} \mathrm{yr}$ as obtained in the standard case. Massive stars emerge from their parental cloud when they have already burnt a sizable fraction of their $\mathrm{H}$-content, etc... Future models are likely to couple the star evolution with that of the accretion disk and of the collapsing cloud. 


\subsubsection{H and He burning phases}

Grids of models of massive stars are currently produced by several groups, at Geneva (cf. Meynet et al., 1994, A\&A 103, 97), at Padova (cf. Fagotto et al., 1994, A\&AS 106, 275), by Stothers and Chin (1996; ApJ 468, 842), by Langer et al. (1994, A\&A 290, 819), by Woosley et al. (1993, ApJ 411, 823; 1995 ApJ $448,315)$ and for binary stars by de Loore \& Vanbeveren (1994, A\&AS 103, 67; 1996, A\&A 305, 825). A database collecting the main stellar models, isochrones and spectral libraries from 1200 to $9800 \AA$ for galaxy evolution modeling has been established recently (cf. Leitherer et al., 1996, PASP 108, 996). As for other mass domains, there are some lingering difficulties concerning the opacities, in particular for the red supergiants and concerning the treatment of convection, semiconvection and overshooting. Most critical in view of the major role of stellar winds are the mass loss rates and their depend ence on $L, T_{\text {eff }}$ and metallicity $\mathrm{Z}$. The rates for supergiants are noticeably uncertain.

A step forward in stellar modeling has been made by Schaerer (1996, A\&A 309, 129), who constructs "complete models", including a simultaneous modeling of stellar interiors atmospheres and winds. These models provide the detailed line spectrum from UV to IR during the course of the evolution.

Among topical results of comparison between models and observations, we notice that the mass discrepancy found by the Munich group between spectroscopic and evolutionary masses was, as shown by Schaerer \& Schmutz (1994, A\&A 288, 23), partly due to the use of plane parallel atmosphere models and partly due to insufficient iron opacities in the atmospheres, as shown by Lanz et al. (1996, ApJ 465, 359). Observations of chemical abundances show that $\mathrm{O}$-stars, rotating faster than $150 \mathrm{~km} / \mathrm{s}$, exhibit $\mathrm{He}$ and N/C enrichments (cf. Herrero 1994, Space Sci. Rev. 66, 137). A number of authors (Fitzpatrick \& Bohannan, 1993 ApJ 404, 734; Venn 1995, ApJS 99, 659; Venn 1996, PASP 108, 309) also find a general evidence of $\mathrm{He}$ and $\mathrm{N}$ enrichments in $\mathrm{B}$ and A-supergiants, a fact which is generally not predicted by standard models. We also note the extended upper main sequence and the absence of the predicted gap in the HR diagram just at the end of the MS. The ratio B/R of blue to red supergiants is also a problem (Langer \& Maeder 1995, A\&A 295, 685), no set of grids of models being able to account simultaneously for the observed B/R at low metallicity Z (like in the SMC) and at high Z (like in the solar neighborhood).

Wolf-Rayet (WR) stars have also gained a major interest owing to the specific problems related to their strong winds and also because they can, due to their emission lines, even be observed in the integrated spectrum of distant galaxies (cf. Maeder \& Conti, 1994, Annual Rev. A\&Ap. 32, 227). WR stars are bare cores, left by mass loss from initially massive stars. Recent abundance determinations for WN stars (with products of $\mathrm{H}$-burning) and WC stars (with products of He-burning) have sharpened the description of their evolutionary status (cf. Crowther et al. 1995, A\&A 297, 172; A\&A 293, 403; A\&A 293, 427; A\&A 302, 457; A\&A 304, 269), (Hamman et al. 1995, A\&A 299; Koesterke \& Hamman, 1995, A\&A 299, 503). For WR stars, a particularly interesting plot is the hydrogen surface contents vs. $\log \mathrm{L} / \mathrm{L}_{\odot}$ (cf. Langer et al. 1994, A\&A 290, 819), while for WC stars the critical plot is $(\mathrm{C}+\mathrm{O}) / \mathrm{He}$ vs. $\log \mathrm{L} / \mathrm{L}_{\odot}$ (cf. Maeder \& Meynet 1994, A\&A 287, 803). The WR/O and WN/WC number ratios are very different from galaxy to galaxy and stellar models, taking into account the $\mathrm{Z}$-dependence of the mass loss rates are able to account for the observed number ratios. In the case of starbursts, the WR/O ratios are much higher and this may be well explained by burst models, assuming very short bursts and ages of the order of a few $10^{6} \mathrm{yr}$ (cf. Meynet 1995, A\&A 298, 767). Thanks to WR stars, one may thus have access to the mass, age, IMF and SFR of distant starbursts.

\subsubsection{Nucleosynthesis}

Very extensive calculations of nucleosynthetic yields have been performed over recent years. Weaver \& Woosley (1995, ApJS 98, 617 and ref. therein) calculated the evolution and nucleosynthesis of models up to the iron core collapse, in the mass range of 11 to $40 \mathrm{M}_{\odot}$ for a broad range of metallicities. The explosive nucleosynthesis is calculated and yields of isotopes lighter than $A=66$ (zinc) are provided. These data were used in models of galactic chemical evolution by Timmes et al. (1996, ApJS 98,617). Nucleosynthesis for models with mass loss was investigated by Woosley et al. (1993, ApJ 411, 823; 1995, ApJ 448, 315; cf. also Maeder 1992, A\&A 264, 105). Other detailed nucleosynthetic calculations were also perf ormed by Thielemann et al. 1996 (ApJ 460, 408 and ref. therein) who pay a particular attention to the location of the "mass cut" between the ejecta and the collapsing core. Various estimates of the mass accretion, due 
to the reimplosion of heavy elements have been made (see also Woosley, ref. above) and the amount of mass accreted by the collapsed core has been found to be of considerable importance. In all calculations, the rate of ${ }^{12} \mathrm{C}(\alpha, \gamma){ }^{16} \mathrm{O}$ reaction always remains a critical parameter.

Despite many progresses, a number of serious nucleosynthetic problems still remain (cf. Langer et al., 1996, 4th Conf. Nuclei in the Cosmos, in press), in particular about the main producers of ${ }^{12} \mathrm{C}$ (intermediate or massive stars?), on the origin of the possible primary nitrogen, on the source of ${ }^{26} \mathrm{Al}$ in the Galaxy, on the site of $\mathrm{r}$ process elements, on the relative ratio $\Delta Y / \Delta Z$ of helium to heavy elements enrichments, etc... Future models of massive stars with rotational mixing may offer attractive perspectives for new nucleosynthetic channels (see §3.4.2).

\subsection{STARS OF INTERMEDIATE MASS}

\subsubsection{Pre-Main Sequence Evolution}

After the major breakthrough which consisted in taking accretion properly into account (Stahler 1988, ApJ 332, 864), the modeling of the PMS phase now addresses the evolution of the rotation rate: The goal is to reproduce the observed distribution of rotational velocities in the youngest clusters. Several processes interfere during this phase: coupling with (and decoupling from) the accretion disk, angular momentum loss through the wind, transport of angular momentum within the star, structural changes.

The dynamical coupling between the convection zone and the radiative interior is often treated in a crude way (Li \& Cameron 1993, MNRAS 261, 766; Bouvier 1995, 8th Cambridge workshop on Cool Stars, Stellar Systems and the Sun, 151; Barnes \& Sofia 1995, ApJ 462, 746; Cameron, Campbell \& Quantrail 1995, A\&A 298, 133). Few papers have dealt explicitly with the internal transport of angular momentum, which they attribute to a specific physical process. Keppens, MacGregor \& Charbonneau (1995, A\&A 294, 469) invoke magnetic torquing, whereas Chaboyer et al. (1995, ApJ 441, 865 \& 876), Krishnamurthi \& Pinsonneault (1997, ApJ, May 1 issue), Matias \& Zahn (1997, IAU Symp. 181) rely on hydrodynamical mechanisms. Chaboyer et al. (1995) take also microscopic diffusion into account, and they evaluate the depletion of lithium.

\subsubsection{Horizontal branch stars}

Up-to-date evolutionary models for stars on the horizontal branch (HB) have been constructed by Yi et al. (1993, ApJ 411, L25) and Lee et al. (1994, ApJ 423, 248), with emphasis on the evolutionary masses of RR Lyrae variables in comparison with their pulsational masses. Constraints on mass loss in the RR Lyrae instability strip based on a comparison of evolutionary tracks with mass loss and observed rates of change of RR Lyrae periods (Koopmann et al. 1994, ApJ 423, 380). The evolutionary status of the stars observed on the extended HB of globular star clusters, whose field counterparts are the sudwarf B stars, has been a long standing problem. Believed to have the structure of ordinary HB stars, i.e. a helium burning core, surrounded by a very low mass hydrogen rich envelope in which hydrogen burns in a thin shell, their low envelope mass cannot be accounted by standard single star stellar evolution. It is possible that the subdwarf B stars are the result of mass transfer in binary systems, or in some cases have been stripped of their envelopes by close interactions with other stars in the dense regions of globular clusters. These stars have received special attention in recent years because of their important contribution to the ultraviolet part of the spectral energy distribution of the integrated spectra of elliptical galaxies. The work of Castellani\& Castellani (1993, ApJ 407, 647) who included mass loss in their evolutionary calculations, shows that rapid mass loss near the tip of the giant branch can affect the mass of the helium core mass at helium ignition, and that ignition can occur when the star has evolved away from the giant branch toward the blue HB. This important result, which may explain the existence of single subdwarf B stars in the field, has been followed up and extended by D'Cruz et al. (1996, ApJ $466,359)$. Another significant advance on the subdwarf stars which should help clarify their internal structure and their evolutionary status, is the discovery that subdwarf B stars are non-radial pulsators. Remarkably, the observational discovery (Kilkenny et al. 1996, MNRAS in press) occurred simultaneously and independently a theoretical prediction of the likely instability of these stars (Charpinet et al.1996, ApJL in press). 


\subsubsection{Globular cluster ages}

The ages of the globular star clusters in the Galaxy are important for understanding the process of formation of the galactic halo and old disk. Research in the last few years has revealed a great complexity in the stellar populations in the galactic halo as in the galactic disk and bulge, and a range in ages among globular clusters. The problem of the relative ages ofglobular clusters has been considered by Chaboyer et al. (1996, ApJ 459, 558). The dating of the oldest globular clusters also sets a lower bound to the age of the universe, and thus is of relevance to cosmology. Recent improvements in the equation of state of stellar matter, which includes a treatment of electrostatic (Coulomb) interactions (OPAL; Rogers et al. $1995,456,902)$ were found by Chaboyer and $\operatorname{Kim}(1995$, ApJ 454, 767) to decrease globular cluster age estimates by about seven per cent (all else being equal). Moreover, VandenBerg et al. (1996, Ann. Rev. A\&Ap 34, 461.) conclude that taking into account atomic diffusion reduces the age of globular clusters by somewhat less than $10 \%$.

In order to evaluate the most likely age for the oldest globular clusters on the basis of our best understanding and to evaluate the magnitude and relative importance of the many sources of errors in globular cluster dating, a Monte Carlo study was carried out by Chaboyer et al. (1996, Science 271, 957). The median age was found to be 14.56 Gyr with a one sided $95 \%$ lower confidence limit of $12.07 \mathrm{Gyr}$.

\subsubsection{Open cluster ages; size of the mixed core}

Open star clusters have long been used to test the theory of stellar structure and to study the chronology of the galactic disk. Now that improved CCD photometry is available, and what is most important in the case of open clusters which are relatively sparse, memberships from proper motion studies, it is possible to perform much more detailed fitting with theoretical isochrones than possible just a few years ago. The size of the mixed core, and the extent of mixing beyond the edge of the convective core as defined formally by the Schwarzschild criterion are particularly significant test of stellar structure. Such studies have recently been carried out for NGC2420 (Demarque et al. 1994, ApJ 426, 165), NGC752, M67 and NGC188 (Dinescu et al. 1995, AJ 109, 2090) and NGC 3680 (Khozurina-Platais et al. 1997, AJ March issue)

\subsection{LOW MASS STARS, BROWN DWARFS, EXOPLANETS}

The last three years have seen a blooming interest in the field of low-mass objects, low-mass stars near the bottom of the main sequence, brown dwarfs and extrasolar giant planets. This interest stems from tremendous progress both in observation and in theory. Ground based and space based surveys, in particular the Hubble telescope which reaches unprecedented faintness and precision, have revealed objects near the bottom of the main sequence, both in clusters and in the Galactic field. From the theoretical view point, recent progress in the description of the microphysics of dense and cool stellar matter, in particular concerning the equation of state, and the elaboration in parallel of synthetic spectra and non-grey atmosphere models have opened the way to evolutionary models which for the first time describe accurately the color-magnitude diagrams of Pop. I and Pop. II M-dwarfs down to the hydrogen burning limit (Baraffe et al. 1995, ApJ 446, L35; also 1997, A\&A).

One of the highlights of stellar astronomy within the past few years is undoubtedly the discovery of two bonafide brown dwarfs: Gliese229B (Oppenheimer et al. 1995, Nature 378, 463) and Teide1 (Rebolo et al. 1995, Nature 377,129 ). The discovery of these objects provides a stringent test for the modelization of both the atmospheres and the interiors of substellar objects. These models allow the determination of the mass of these brown dwarfs, namely $0.02-0.05 \mathrm{M}_{\odot}$, well below the hydrogen-burning limit (Allard et al. 1996, ApJ 465, L123; Marley et al. 1996, Science 272, 1919).

At last the search for the existence of substellar objects has been rewarded by the recent discovery of several extrasolar giant planets (Mayor \& Queloz 1995, Nature 378, 355; Butler \& Marcy 1996, ApJ 464, L53). This opens a new field of astronomy, both for observers and theorists, since exoplanets should be in reach of several future observational projects.

Besides the intrinsic interest of low-mass objects from the point of view of basic physics and stellar 
evolution theory, these objects represent plausible candidates for the existence of dark baryonic matter in the galactic disks and halos. The combination of standard astronomy, i.e. star counts, for the visible part, and of microlensing experiments for the substellar contribution, will allow soon the precise determination of the contribution of these objects to the Galactic mass budget, and to the baryonic fraction of the Galactic missing mass, which remains one of most important problems in astrophysics.

\subsection{DIFFUSION AND MIXING IN RADIATIVE INTERIORS}

\subsubsection{Atomic diffusion}

The importance of the role of atomic diffusion in stellar evolution has been confirmed by helioseismology measurements. Over the last three years Proffitt (1994, ApJ 425, 849), Chaboyer et al. (1995, ApJ 446, 435), Richard et al. (1996, A\&A 312, 1000), Basu et al. (1996, ApJ 460, 1064) Guenther et al. (1996, ApJ 463, 382), Bahcall et al. (1996, PRL, submitted), have all concluded that atomic diffusion considerably improved the agreement between the observed and calculated pulsation spectra of the Sun. The OPAL equation of state also appears to improve the agreement especially for frequencies influenced by the convection zone (Bahcall et al. 1996). There are indications from low 1 measurements that atomic diffusion may play a role in the central solar regions (Basu et al. 1996). The He settling suggested by helioseismology is furthermore consistent, within the uncertainty of the models, with the turbulence needed to explain the solar Li underabundance (Proffitt \& Michaud 1991, ApJ 380, 238). These results suggest that atomic diffusion probably plays a role in the evolution of most stars, even though some times small, and that it should be included in "standard stellar evolution".

It has been suggested that diffusion plays a major role in A and B stars to explain the large abundance anomalies that have been observed in them (Michaud 1970, ApJ 160,641). However it also appears that mass loss competes with atomic diffusion in most stars with abundance anomalies (see e.g. Vauclair 1975, A\&A 45, 233; Michaud et al. 1983, ApJ, 269, 239). Babel (1995, A\&A, 301, 823; 1996, A\&A, 309, 867) has done quantitative estimates of the mass loss rates driven by radiative winds in these objects. He reproduces well the $T_{\text {eff }}, \log g$ interval where abundance anomalies are to be expected. It remains to couple such mass loss models to evolutionary models taking into account diffusion of atomic species in order to arrive at more quantitative comparisons with observed abundance anomalies.

\subsubsection{Rotational mixing}

There are some indications that rotating stars experience a limited amount of mixing in their radiative interior. Among massive stars, the rapid rotators show a significant enhancement of their ${ }^{4} \mathrm{He}$ surface abundance (Herrero et al. 1992, A \& A 261, 209), and in solar-mass stars the destruction of ${ }^{7} \mathrm{Li}$ seems to be correlated, partly at least, with the loss of angular momentum through the wind (Soderblom et al. 1993, AJ 106, 1059), as confirmed by the underdepletion of tidally-locked binaries (Thorburn et al. 1993, ApJ 415, 150; Ryan \& Deliyannis 1995, ApJ 453 , 819; Zahn 1994, A\&A 288, 829). It is thus natural to verify whether the same physical mechanism can be responsible both for the internal transport of angular momentum and that of chemical elements. Two physical processes are known to have that property, i.e. the thermally driven meridian circulation and the shear instabilities induced by differential rotation, and they have now been implemented into several stellar evolution codes, with various degrees of sophistication.

The results obtained so far with massive stars are encouraging (Denissenkov 1994, A\&A 287, 113; Urpin et al. 1996, A\&A 306, 455; Fliegner et al. 1996, A\&A 308, L13; Talon et al. 1997, A\&A in press), although it is hard to distinguish between the consequences of convective penetration, semi-convection and rotational mixing. Another problem is the build-up of molecular weight gradients, as evolution proceeds. In general, they strongly inhibit the mixing (Meynet \& Maeder 1996, A\&A in press), but their stabilizing effect can be weakend through the anisotropic turbulence produced by the differential rotation in latitude (Maeder 1996, A\&A in press; Talon \& Zahn 1996, A\&A in press).

The internal mixing occurring in late-type stars continues to receive much attention, because it is of crucial importance for the determination of the original lithium abundance, which provides one of the 
few observational constraints on the big bang nucleosynthesis. Evolutionary sequences with rotational mixing have been built by Pinsonneault et al. (1989, ApJ 338, 424; 1990, ApJS 74, 501), Charbonnel et al. (1992, A\&A 255, 191), Chaboyer et al. (1995, ApJ 441, 865); their results on the Li abundance in Pop. I stars are in reasonable agreement with the observations, although it may be argued that they involve one or several adjustable parameters. The application to halo stars suggests that rotational mixing has depleted some Li during their evolution (Chaboyer \& Demarque 1994, ApJ 433, 510).

However the steep rotation profile which is predicted in the solar interior by such calculations is incompatible with the flat profile deduced from helioseismic inversions (cf. §3.6). Recent improvements brought to the treatment of rotational mixing (Zahn 1992, A\&A 265, 115) even worsen the disagreement (Matias \& Zahn 1996, IAU Symp. 181).

\subsubsection{Internal waves}

It thus appears that another, more powerful mechanism is responsible for the transport of angular momentum in the Sun, and hence also in other stars. One candidate has been recently examined, namely the transport by the internal gravity waves emitted at the base of the convection zone. According to the first and rather crude estimates, this mechanism reaches easily the required efficiency (Kumar \& Quataert 1997, ApJ in press; Zahn et al. 1997, A\&A in press). It remains to be seen how this powerful process will interfere with the rotational mixing investigated so far. The same internal waves may also directly produce some mixing, as has been suggested by Press (1981, ApJ 245, 286) and more recently by Schatzman (1993, A\&A 279, 431; 1996, J. Fluid Mech. 322, 355); work is in progress to confirm the validity of this mechanism.

\subsection{CONVECTION}

The past three years have seen rapid progress in fully numerical simulations of convection near stellar surfaces. The $a b$ initio simulations by Nordlund, Stein and collaborators (in which the only input is the atomic physics of partially ionized gases and the equations of radiative transport and hydrodynamics) had already achieved very close agreement with observations of the Sun in previous reporting periods, in particular in variables such as the average heat flux, the details of the line profiles and the morphology and time evolution of the granulation. Recent work at higher numerical resolution (Nordlund and Stein, 1995 in the $32^{\text {nd }}$ Liège Colloquium, ed. A. Noels et al.; Nordlund et al. 1996 Bull. Astron. Soc. India 24, 261) now shows granulation patterns that are nearly indistinguishable from real granulation, quantitatively or by the human eye. Preliminary analysis of the excitation of p-modes and the effect of convection on their frequencies also shows substantial agreement with observations. The early conclusions from this work have now been confirmed independently by other groups (Ludwig et al. 1994, A\&A 284, 105; Solanki et al. 1996, A\&A 308, 623; Atroschenko and Gadun 1994, A\&A 291, 635).

Building upon this success, Ludwig et al. (in $32^{\text {nd }}$ Liège Colloquium) have begun an extensive program to simulate stellar surface convection at different locations in the HRD. These results can be used to calibrate the mixing-length to scale height ratio $\alpha$ used in stellar structure calculations. For envelopes on the giant branch, the main source of uncertainty currently seems to lie in the treatment of the low-temperature atmospheric absorption coefficients, both internally in a given simulation and in the comparison with mixing length models.

The main physical insight from the convection simulations is that the heat transport near a stellar surface is nonlocal in an extreme way: the entropy fluctuations seen at any depth are dominated by advection of cold material from the stellar surface, rather than by local mixing processes (as seen also in labor atory experiments at high Rayleigh number). This is likely to have consequences for many processes which are traditionally modeled with a local mixing-length picture (some of these consequences have been explored by Spruit, Mem. Soc. Astron. It., 1997, in press).

Direct numerical simulations in three dimensions show that in the bulk of a convective region the downwards directed motions are more vigorous, more concentrated and that they may span several scale-heights (Brummel et al. 1995, Science 269, 5229; Porter et al. 1995, Comput. Appl. Math. 14, 97). However the 
fluxes in these downflowing plumes partly cancel, leaving the quieter upflows to carry about $2 / 3$ of the convective flux. Other simulations, performed with a sub-grid scale prescription, give similar results (Kim and al. 1995, ApJ 442, 422; 1996, ApJ 461, 499).

Work on more traditional convection formulations based on turbulence models has been pursued by Canuto and collaborators and by Grossman. A 'parameter free' local turbulence model (1991, ApJ 370, 295) claimed to reproduce the solar radius at $0.5 \%$ accuracy was found to need introduction of adjustable parameters when newer opacity tables were used. Thus, the theory needs calibration by observations or numerical simulations (this also applies to the further developments of the theory in ApJ 389, 724; ApJ 467,385 ). Other parametrizations, also inspired by numerical simulations, have been explored by Lydon et al. (1993, ApJ 403, L79; ApJ 413, 390). Grossman (1993, ApJ 407, 284; 1996, MNRAS 279, 305) has developed the most elaborate theories to date in which transport of various fluctuation variables is modeled by second order (diffusion type) equations.

There is still no explanation for the apparent lack of convective penetration at the base of the solar convection zone, as revealed through acoustic sounding (see below). This is surprising, since laboratory experiments and direct numerical simulations (Hurlburt et al. 1994, ApJ 421, 425; Mutsham et al. 1995, A\&A 293, 127) all display substantial penetration. However the rotation, whose role has been neglected so far, seems to have an inhibiting effect on penetrative convection (Julien et al. 1996, Dynam. Atmosph. $\&$ Oceans 24, 237).

\subsection{HELIOSEISMIC CONSTRAINTS}

Helioseismic techniques have made a tremendous contribution to our knowledge of the Sun over the past. few years. The quality of the available helioseism ic data has improved significantly, thanks to instruments like LOWL and networks like BiSON, IRIS and GONG and the SOHO space mission. These have allowed us to determine the structure and rotation rate inside the Sun to very high precision, with errors in the sound-speed determination of only a few part in $10^{4}$. Because of the seismic inferences, the standard solar model has improved considerably (Christensen-Dalsgaard et al. 1996, Science 272, 1286) through improvements in input microphysics. What we know so far suggests that the internal structure of the Sun can be represented to a good approximation by a standard solar model, however, one which has a smoother sound speed and abundance profiles than the solar models with the usual treatments of diffusion and settling.

Perhaps the single most important result in the past few years is the firm establishment of the importance of diffusion and gravitational settling of helium and heavy elements below the solar convection zone. While the relative difference between the sound-speed profile of the Sun and solar models with settling is less than $0.25 \%$, for models without settling the difference is as large as $1 \%$ (Basu et al. 1996, ApJ 460, 1024, Bull. Astron. Soc. India 24, 147).

The sound-speed inversion results show that models with usual treatments of diffusion have a lower sound-speed than the Sun immediately below the solar convection zone. In fact, all models which have sharp change in their composition profiles below the solar CZ have been found to be inconsistent with observations (Basu \& Antia 1994, MNRAS 269, 1137). This indicates the need for some mixing below the solar CZ (Gough et al. 1996, Science 272, 1296; Kosovichev et al. 1997, Sol. Phys. in press). However, evidence also suggests that there is very little, if any, classical overshoot below the solar convection zone (Monteiro et al. 1994, A\&A 283, 247; Basu \& Antia 1994, MNRAS 269, 1137). Models with mixing induced by rotation (Chaboyer et al. 1995, ApJ 446, 435; Richard et al. 1996, A\&A 312, 1000) seem to satisfy observed sound-speed and lithium abundance constraints.

Helioseismic measurements of helium abundance indicate that the the helium content in the solar convection zone is in the range 23-25\% (Pérez Hernández \& Christensen-Dalsgaard 1994, MNRAS 267, 111; Basu \& Antia 1995, MNRAS 276, 1402; Kosovichev 1996, Bull. Astron. Soc. India 24, 355). This is an indirect evidence for diffusion below the solar CZ. In absence of diffusion a helium abundance of $27-28 \%$ is required in the solar envelope to satisfy solar luminosity constraint; the observed value can be explained only if helium has settled out of the CZ. In the absence of diffusion the observed helium abundance is also inconsistent with the primordial helium abundance as obtained by standard cosmological models. 
The Sun has also been successfully used as a laboratory to test microphysics like the equation of state and opacity of the solar material. From inverted sound-speed differences between the Sun and models it can be shown that the OPAL equation of state is a better representation of the solar equation of state than the MHD equation of state (Basu \& Antia 1995, MNRAS 276, 1402; Basu et al., 1996, Bull. Astron. Soc. India 24, 147).

Helioseismic data along with the equations of thermal equilibrium can be used to study the thermal structure. It is found that if the opacities of thesolar material are not substantially different from the OPAL opacities, then the central temperature of the Sun is about $15.7 \times 10^{6} \mathrm{~K}$ and the predicted neutrino fluxes are significantly higher than the observed values unless large changes in opacities are allowed (Antia \& Chitre 1995, ApJ 442, 434; 1996, Bull. Astron. Soc. India 24, 321).

In the field of solar rotation, the early results which showed that the Sun rotates differentially in the convection zone but almost like a solid body in the radiative interior (Christensen-Dalsgaard \& Schou 1988, Proc. Symp. Seismology of the Sun and Sun like stars, ed. Rolfe, ESA SP-286, p 149; Rhodes et al. ibid., p73) have been re-confirmed (Thompson et al. 1996, Science 272, 1300). The change in the nature of the rotation rate takes place slightly below the base of the convection zone (Kosovichev 1996, ApJ 469, L61). There is also a shear layer just below the surface, where the rotation rate increases with depth. Whether the solar core rotates faster or slower than the surface is still a matter of debate (Elsworth et al. 1995, Nature 376, 669; Lazrek et al. 1996, Sol. Phys. 166, 1).

New helioseismic inversion techniques have been increasingly used over the last few years to study localized regions. The technique of ring-diagrams (Hill 1988, ApJ 333, 996) allows to track horizontal flows (Pátron et al. 1995, ApJ 455, 746). Time-distance helioseismology (Duvall et al. 1996, Nature 379, 235) has also been used to probe the upper convection zone (Kosovichev 1996, ApJ 461, 155) and reveal large scale sub-surface structures and flows related to the active regions.

\subsection{SOLAR NEUTRINOS}

A large number of papers concerning the solar neutrinos have been published in the last few years. They are of three different types: (1) data from the three experiments presently active, Homestake, Kamiokande and Gallex and discussions about the meaning of these data; (2) solar models with predictions of the neutrino flux; and (3) theories of the solar neutrino deficiency. Many papers of

the latter kind are highly specialized in theoretical physics and we shall not consider them in this review.

Davis lectured on the Homestake solar neutrino experiment (1994, International school of Nuclear Physics, 15th Course, ed. Faessler, Elsevier) and Haxton wrote a complete review on the subject, including the point of view of physicists explaining the neutrino flux deficiency (1995, Annual Rev. Astr. Astrophys 33, 459). The first Gallex results (Gallex I+II) gave a flux of 77.1 \pm 9 SNU (1995, Phys. Letters B 357, 237), whereas the last results (Gallex III) are significantly lower: $53.9 \pm 11$ SNU (Hampel et al. 1996, Phys. Letters B 388, 384); the average of the three Gallex runs amounts to $69.7 \pm 8 \mathrm{SNU}$. Other recent references are given in this last paper, and also a discussion of the calibrations by the ${ }^{51} \mathrm{Cr}$ source. Note also the account of the Gallex experiment given by Kirsten (1995, Ann. N. Y. Acad. Sci. 759). The "Kamiokande solar neutrinos results" are collected in Nucl. Phys. B Proc. Suppl. (38, 54-59, 1995), and by the time these Transactions appear, we should know the level measured by Super-Kamiokande. The question of the time variation of the neutrino flux is approached in several papers: Gavryusev \& Gavryuseva (1994, A\&A 283, 978), Liritzis (1995, Solar Physics 161, 29), Mc Nutt Jr. (1995, Science 270, 5242), Vasil'ev and Ogartsov (1995, Astron. Lett. 21, 491).

Solar models lead still to predictions of the solar neutrino flux which are consistently larger than the observed ones, and the discrepancy depends on the detection threshold of the experiment. Dzitko et al. (1995, ApJ. 447, 428) discuss in great detail the physical problems involved. The so-called Standard Solar Model (SSM) now includes the atomic diffusion of helium and the heavy elements (Bahcall et al. 1995, Rev. Mod. Phys. 67, 781); it implies a completely quiet radiative interior, which would not as such explain the observed Li depletion, and therefore it cannot be considered as definitive. Similar models have been built by Berthomieu et al. (1995, ASP conf. ser. 76, 109). Solar Modeling (1996, ed. Balantekin \& 
Bahcall, World Scientific) collects twenty papers discussing solar models from different points of view. An important article by Dar \& Shaviv (to be published in ApJ) initiates a new discussion of the validity of various physical assumptions. Bahcall (1996, Rosseland Centenary Symposium, Frontiers of Astrophysics, 151) presents "Two solar neutrino problems", in the frame of SSM. By constraining models of the central regions of the Sun, helioseismology has important input in discussions of the solar neutr ino fluxes (see e.g. Chaboyer et al. 1995, ApJ 446, 435; Guenther et al. 1996, ApJ 463, 382).

A variety of physical problems have been considered, to explain the neutrino flux deficit: Coulomb dissociation of ${ }^{8} \mathrm{~B}$ by Gai (1995, Nucl. Phys. B. Proc. Suppl. 38, 71); uncertainties about the ${ }^{71}$ Ga capture cross section by Hata \& Haxton (1995, Phys. Lett. B 353, 422); influence of radiative corrections on the $\beta$-decay process responsible for the production of neutrinos by Batkin and Sundaresan (1995, Phys. Rev. D. 52, 5362); influence of a third body on the rate of nuclear reactions by Batkin and Sundaresan (1995, Phys. Rev. E. 52, 940); the photodissociation of ${ }^{8} \mathrm{~B}$ and the solar neutrino problem by Bertulani (1995, Nucl. Phys. B. 587, 318); condensed matter effects (N-body problem) for the $p\left(p, e^{+} \nu\right) \mathrm{D}$ and ${ }^{8} \mathrm{~B}$ solar neutrino flux by Kim et al. (1996, AIP Conf. Proc. 334, 995); electrostatic screening of nuclear reactions by Shaviv and Shaviv (1996, ApJ. 468, 433); plasma effect in the solar core and the solar neutrino problem by Tsytovitch et al. (1996, Astroparticle Physics 5, 197); improvements of nuclear physics input by Kim et al. (1995, Nucl. Phys. B. Proc. Suppl. 38, 293).

To conclude, it is still impossible to guess whether this long standing problem will be solved by the astrophysicists or by the particle physicists, or by both. 